\title{
Phase Contrast Imaging in Thick, Heterogeneous Samples via S-Matrix Phase Retrieval and Depth Sectioning
}

Philipp Pelz ${ }^{1}$, Hamish Brown ${ }^{2}$, Scott Findlay $^{3}$, Mary Scott $^{2}$, Jim Ciston $^{2}$ and Colin Ophus ${ }^{2}$

${ }^{1}$ University of California Berkeley, Berkeley, California, United States, ${ }^{2}$ Lawrence Berkeley National Laboratory, Berkeley, California, United States, ${ }^{3}$ Monash University, Clayton, Victoria, Australia

Three-dimensional phase-contrast imaging of thick, multiply scattering samples in electron microscopy requires the inversion of the paraxial Schroedinger equation for fast electrons which describes the propagation of coherent high-energy electrons in an electrostatic potential. The most prominent method for calculating a coherent exit-wave from a multiple-scattering sample is the multi-slice algorithm [1]. Due to its popularity for forward calculations, the inverse multi-slice algorithm has mostly been used in algorithms for inverting the scattering process, to retrieve a sample structure from measured intensities in real $[2,4]$ or Fourier space $[3,4,5]$.

An alternative way of solving the Schroedinger equation is the scattering matrix (S-matrix) formalism, which is traditionally only used for single crystals due to its computational and memory requirements. Recently, Ophus [6] developed a fast hybrid algorithm to calculate 4D-STEM intensities from a planewave reciprocal-space interpolated scattering matrix (PRISM), which alleviates the memory and computational requirements by allowing the S-matrix and the diffraction intensities to be sampled on different grids. Findlay [8] has proposed an inversion procedure for the scattering matrix of a single crystal from a focal series of 4D-STEM measurements, which was recently experimentally demonstrated [9].

Here we combine the principle of phase-shifting interferometry used in the PRISM algorithm to calculate exit-waves from the S-matrix, and the overlap constraint known from ptychographic reconstruction algorithms to retrieve the phases and amplitudes of the S-matrix of heterogeneous samples. Our algorithm relaxes the requirement of a single-crystalline sample and enables future refinements of probe aberrations and scan positions, as known from ptychographic reconstruction algorithms.

Three-dimensional depth sections can be reconstructed from the retrieved S-matrix [7] by coherently summing all beams after removal of propagation and parallax effects. Other than ptychographic depth sectioning by Wigner Distribution Deconvolution, S-matrix depth sectioning allows applying arbitrary complex weights to each beam before re-interfering them, enabling novel imaging modalities that are cumbersome to realize experimentally.

Fig 1 a) Show a simulated a 4D-STEM focal series experiment at 300keV electron energy and with a convergence angle of $30 \mathrm{mrad}$ to recover the $\mathrm{S}$-matrix of a hypothetical $12 \mathrm{~nm}$ diameter strained $\mathrm{Mn}_{3} \mathrm{O}_{4}-$ $\mathrm{Co}_{3} \mathrm{O}_{4}$ core-shell nanoparticle on an amorphous carbon substrate. For a range of defocus values with focus planes inside the specimen, 4D-STEM datasets are recorded. Fig. 1 b) shows one iteration of generalized S-matrix retrieval from 4D-STEM focal series data.

Fig. 2 a) shows the full 3D atomic structure of the same $\mathrm{Mn}_{3} \mathrm{O}_{4}-\mathrm{Co}_{3} \mathrm{O}_{4}$ core-shell nanoparticle. Fig. 2 b)d) show phase-contrast images take from depth sections of the S-Matrix reconstructed with the algorithm shown in Fig. 1b), taken at different heights within the sample. In Fig. 2 e)-g) we synthesize phase-contrast images of a virtual experiment with hollow-cone illumination by only using the beams included within 
28-30mrad. This suppresses out-of-focus contributions in the phase-contrast images more strongly and makes it easier to identify atomic positions.

In this talk we will also discuss progress towards the experimental demonstration [10].
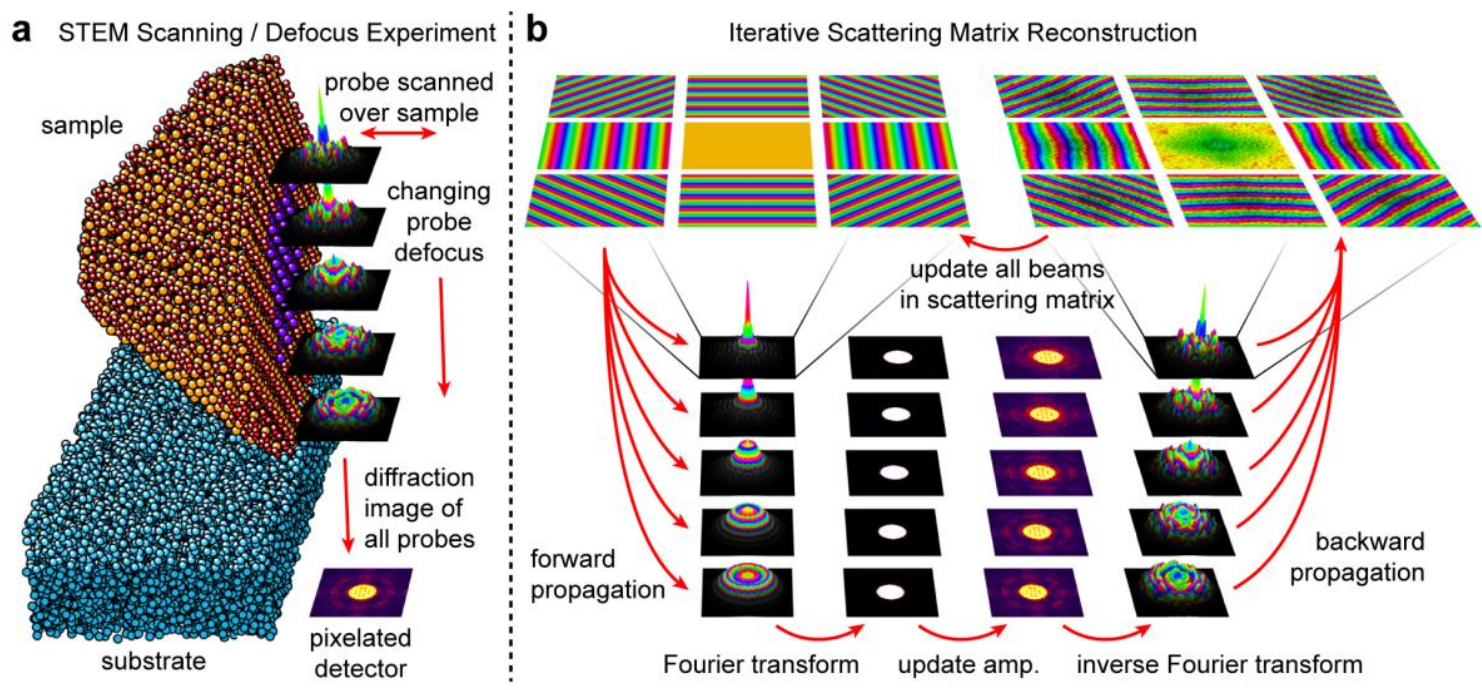

Figure 1. a) sketch of a 4D-STEM focal series experiment. A convergent beam is focused at 5 different focal planes within the sample. b) One iteration of the iterative $S$-matrix retrieval algorithm. All beams of the S-matrix interfere to create 4D-STEM exit waves. The amplitude of the exit waves is updated in Fourier space according to the focal series measurements. Then the aberrations are removed and exit waves are projected back on the S-matrix beams. 


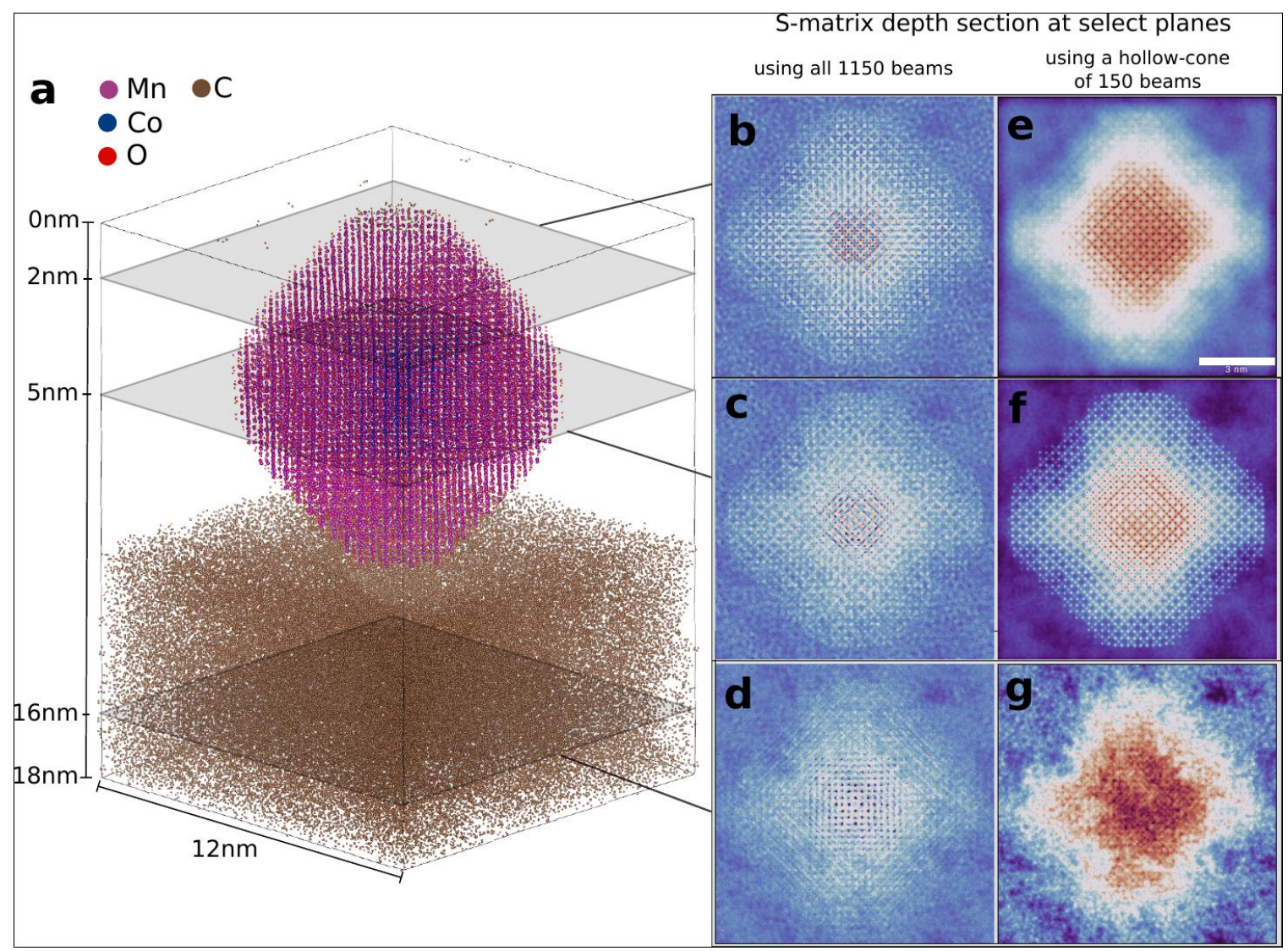

Figure 2. a) 3D structure of a hypothetical 12nm diameter strained Mn3O4-Co3O4 core-shell nanoparticle on amorphous carbon substrate imaged with a 30mrad convergence angle at 300keV electron energy. b), c) and d) Phase of S-matrix depth sections from simulated input data at depths of $2 \mathrm{~nm}$, $5 \mathrm{~nm}$ and $16 \mathrm{~nm}$ within the sample with all 1150 beams in the $30 \mathrm{mrad}$ aperture. e), f), g) Phase of S-matrix depth sections at depths of $2 \mathrm{~nm}, 5 \mathrm{~nm}$, and $16 \mathrm{~nm}$ from a hollow cone of 150 beams from 28-30mrad convergence angle. Out-of-focus contributions are strongly reduced, even more so in the hollow-cone depth section.

\section{References}

[1] Cowley, J. M. \& Moodie, A. F. Acta Crystallographica 10, 609-619 (1957).

[2] Ren, D., et al. Ultramicroscopy 208, 112860 (2020).

[3] Maiden, A. M. et al. J. Opt. Soc. Am. A. Opt. Image Sci. Vis. 29, 1606-14 (2012).

[4] Van den Broek et al. Phys. Rev. B 87, 184108 (2013).

[5] Gao, S. et al. Nature Communications 8, (2017).

[6] Ophus, C. Adv Struct Chem Imag 3, 13 (2017).

[7] Ophus, C. et al. Microscopy and Microanalysis 25, 10-11 (2019).

[8] S. D. Findlay, Acta Crystallogr. Sect. A 61 (2005) 397.

[9] H. G. Brown et al., Phys. Rev. Lett. 121 (2018) 266102.

[10] Work at the Molecular Foundry was supported by the Office of Science, Office of Basic Energy Sciences, of the U.S. Department of Energy under Contract No. DE-AC02-05CH11231. CO acknowledges additional support from the U.S. Department of Energy Early Career Research Program. HGB and JC acknowledge additional support from the Presidential Early Career Award for Scientists and Engineers (PECASE) through the U.S. Department of Energy. M. S. and P. P. acknowledge support from the STROBE NSF Science and Technology Center on Real-Time Functional Imaging. 